\title{
Pathological effects of the renin-angiotensin system on cardiovascular disease
}

\author{
Yan ZHANG \\ Luohe Medical College \\ Luohe,462000, China
}

\begin{abstract}
RAS) found in a research as a new member, changes the past view that only the angiotensin (Ang) II catalyzed by angiotensin-converting enzyme (ACE) can produce pathogenic role. The research proved the renin binding with former prime renin -receptor (RPR) and Ang IV binding with insulin receptor regulated aminopeptidase (IRAP) will also produce the same pathogenic role. The newly discovered ACE2 catalyzes Ang- (1-7) and Ang- (1-9) generation, then the receptor of Ang- (1-7) and Mas and the receptor of Ang- (1-9) and AT2 binding, may produce cardiovascular protective effect, but the pathogenic role of RAS is still dominant. The discovery of RAS new member provides a new theoretical basis to recognize RAS inhibitors in clinical research results.
\end{abstract}

Keywords- renin - angiotensin system; angiotensinconverting enzyme 2; cardiovascular disease

\section{INTRODUCTION}

RAS mainly composed by the renin, angiotensinconverting (angiotensin converting enzyme, ACE) and angiotensinogen can generate angiotensin to exert physiological effects. Renin is a glycoprotein which is a proteolytic enzyme produced and secreted by next ball kidney cells. It also has been found in other tissues (such as the brain, heart, blood vessels, etc.) that the original transition is from the former renin.

ACE is a glycoprotein, composed by a single peptide chain, and zinc atoms. It presents in most tissues of the human heart, blood vessels, kidney, lung, brain, etc. Angiotensin, originally a globulin, produced by the liver, is the substrate for the renin role. It is composed by two peptide chains, and its precursor molecule 24 to 34 amino acid fragments are angiotensin I (angiotensin I, Ang I ).

ACE will degrade Ang I with 10 peptide chains into angiotensin with 8 peptide chains, meanwhile also catabolizes a variety of important biological ACE peptides (e.g. substance P, bradykinin) into catabolism inactive product. Ang II becomes inactive by enzymatic hydrolysis of angiotensin; Ang II can also be hydrolyzed by aminopeptidase, generating angiotensin III (angiotensin III, Ang III). There is also another angiotensin (1-7), with the effect of vasodilator and lowering blood pressure. Besides, Ang II is the most important biological systems effector molecules. It has both systems hormone (endocrine) effects, but also the local production factor (paracrine, autocrine) effect.

\section{PHYSIOLOGICAL ROLE OF RENIN - ANGIOTENSIN SYSTEM}

Ang II : Ang II is currently one of the most potent known vasoconstrictor substance, and its blood vessels is a direct vascular smooth muscle contraction, rather than vasoconstriction by adrenergic mechanisms. Experiments show that, ion dynamics foundation of Ang II vasoconstrictor effect is making $\mathrm{Ca} 2+$ into vascular smooth muscle cells. Ang II having positive inotropic effect on ventricular skin, intravenous Ang II, can increase cardiac output and heart rate. Ang II also binding with presynaptic angiotensin receptor on sympathetic vasoconstrictor fiber tip plays presynaptic regulatory role, so norepinephrine (NE) released by sympathetic nerve endings increases to strengthen the cardiovascular stimulant.

Ang II receptors: No matter which way by forming Ang II, its physiological role must be combined with the highly specific receptor in order to play. According to the receptor sites in the cell, it can be divided into two categories, the cell membrane receptor and the receptor cell nucleus and chromosomes. Ang II cell surface receptors is the main mediator of Ang II function. Ang II receptors are widely distributed in the heart, blood vessels, liver, kidneys, lungs, gastrointestinal tract and adrenal substandard peripheral organs. Ang II, the cells made on its own, through the way of cellular endocrine synthesis mediates its physiological function by Ang II specific binding sites on the cell nucleus and chromosomes. Especially Ang II in cardiovascular local organizations can be synthesize by DNA promoting vascular smooth muscle cells and protein, causing vascular smooth muscle cells causing hypertrophy. In 1994, the International Union pharmacology (the International Union of Pharmacology, IUPHAR) angiotensin receptor nomenclature committee passed the latest nomenclature of the angiotensin receptor: the use of angiotensin receptor abbreviation is AT; AT receptor subtypes in the AT Note below to 1,2,3,4, etc. Receptors which are antagonistic to losartan or similar substance is AT1,receptors being antagonistic to PD123177 or the like receptor is AT2; AT1 or AT2 receptor further subdivided, then using the $\mathrm{A}, \mathrm{B}, \mathrm{C}$ represent. In cardiac myocytes and non-myocytes there are AT1 receptors. AT1 receptors belong to the $\mathrm{G}$ protein-coupled receptor superfamily, which is composed by 359 amino acids, a 
relative molecular weight of $41 \times 103$, and has seven transmembrane domains on the family structure. By $G$ protein-coupled, and phosphatase A, C and D, phosphatidylinositol, and calcium channel serine / threonine, tyrosine kinases and other signal transduction pathways linked.AT1 receptors dominate in body fluids - the dominant expression and electrolyte balance and blood pressure regulation in tissues and organs, therefore, it is mainly located in the adrenal glands, vascular smooth muscle cells. It seems that in mature tissues all known Ang II effect is mediated by AT1 receptors, including: Inotropic effect of myocardial tissue stimulation of cell growth and positive change, stimulating the proliferation of vascular smooth muscle cells, vascular smooth muscle contraction, and stimulation to increase sympathetic neurotransmitter release, stimulating the secretion of vasopressin and aldosterone release, controlling water intake and urinary sodium excretion. Ang II binding with AT1 receptor can mediate cardiomyocyte and fibroblast hyperplasia and proliferation, and contribute to cardiovascular remodeling.

\section{THE RELATIONSHIP BETWEEN ACE2'S AND CARDIOVASCULAR DISEASE}

Influence of ACE2 on blood pressure: cause of essential hypertension is multifactorial, including the interaction of environmental factors and genetic factors. RAS system is the major player in blood pressure homeostasis, and significantly affects the pathophysiology of hypertension. Studies have shown that, ACE2 may be a negative regulator of the RAS system, which has an impact on blood pressure and by the interaction of RAS and other regulatory peptides.

The study found that in rat kidney hypertension, the protein level of ACE2mRNA and ACE2 significantly decreased, and was negatively associated with high blood pressure. Relative analysis showed that the level SS-X QTL with salt-sensitive rats, spontaneously hypertensive rats BP3 QTL, spontaneously hypertensive rats BBXsQTL tendency of stroke overlap ACE2 gene revealed that a point $\mathrm{x}$ chromosome is the candidate gene. At the same time, mapping chromosome location of several quantitative trait loci (quantitative trait locus, QTL) controlling hypertension susceptibility has been identified. The possible mechanism ACE2 on blood pressure regulation, mainly considers that the ACE2 degrades AngII and increases Ang (1-7) generation to participate in the regulation of blood pressure. Recently, however, it was also reported that essential hypertension seems no obvious association with ACE2. Therefore, the exact molecular mechanism of blood pressure regulation on ACE2 remains unclear, pending further exploration and research. The impact Crackower ACE2 on cardiac function by methods such as gene knockout is confirmed from different aspects ACE2, which is a crucial regulator of cardiac function. First, they knockout ace 2 gene of mice (ace 2 - / - mice), direct observing whether ACE2 plays an important role in cardiovascular disease process, and found that the absence of ACE2 can affect cardiac function, left ventricular slightly thinner, myocardial contractility extremely weakened, and found a large number of hibernating myocardium and cardiac suppression Dayton. Meanwhile, the fact hypoxia genes BNIP-3 and PAI1regulated up, indicated missing ACE2 may shrink by coronary microcirculation dysfunction, coronary blood shunt caused by reduction of myocardial blood supply of nutrients, and then dropped like myocardial hibernation / stunning systolic function appears above results suggest that ACE2 may be mediated deletion of myocardial ischemia and hypoxia. According to reports, $28 \mathrm{~d}$ after coronary artery ligation, myocardial expression ACE2mRNA increased about 3 times, suggesting that ACE2 with improved myocardial contractile function, and in the bodies of the mice with ACE2 knockout AngII levels was found elevated, suggesting that ACE2 may confront the role of AngII, thus weakening its mediated cell proliferation and promoting myocardial fibrosis and other adverse effects. The study also found that when the level of the heart failure ACE2mRNA increased significantly, while it's hydrolyzed Ang (1-7) in heart failure patients with ventricular highly aggregate. When primary cardiomyopathy occurred, activity of cardiac ACE2 also showed increased. ACE2 involved in these studies are suggestive of a change in heart function and in which play an important role in the pathophysiology.

Through further study found, $G$ protein-coupled receptor Mas is Ang (1-7) functional receptor with high affinity, whereas the specific molecular mechanisms of heart failure that ACE2 plays a relevant role in the process of needs further study, but at least it can be prompted by increasing ACE2 activity can be improved cardiac function.

The relationship between ACE2 and atherosclerosis: Atherosclerosis is closely related to vascular endothelial injury. Study found that ACE2 null mice, Ang II levels in plasma and cardiac increased. Ang II stimulates endothelial cells and vascular smooth muscle cell proliferation and reduced nicotinamide-adenine dinucleotide phosphate oxidase, and then breaks down and produces endothelial cell NO peroxynitrite, thus weakening blood vessels diastolic function. Studies have shown that level of Ang (1-7) in plasma decreasing and the potential protective effects of vascular endothelium may strengthen the role of the damage AngII on impaired endothelial. In summary, due to the reduction in ACE2 can elevate AngII and reduce Ang (1-7), which will exacerbate the formation of endothelial damage and arterial plaque.

The relationship between ACE2 and arrhythmia: In recent years, the study found that for ACE2, ACE2 and its products Ang (1-7) have antagonized many AngII mediated cardiovascular pathophysiological role, but studies show that ACE2 overexpression of rhythm disorders affecting the performance of a negative effect. By studying high expression in transgenic mice cardiomyocytes ACE2 found, increasing ACE2 protein expression levels, can lead to severe heart block; sustain ventricular tachycardia and ventricular fibrillation fatal lethal ventricular arrhythmias. Using electrophysiological high expression of mouse ACE2 inspection, analysis results, probably due to overexpression of ACE2 in a mechanism such that connexin CX40 and CX43 downregulated, result in a gap cell connection disorders, and ultimately lead to cardiac electrophysiology 
physiological disorders. Meanwhile, transgenic mice with no severe arrhythmia was found, due to the effect of autologous some mechanism can be automatically reduced ACE2 gene, thus restoring the normal conduction, rhythm and connexin expression. Although ACE2 gene transfer research results in mice cannot ultimately establish a direct relationship between the role of ACE2 and cardiac conduction, at least suggestive of ACE2 in the process may open up a new path for the treatment of lethal arrhythmias.

\section{THE EFFECTS AND MECHANISM ANGIOTENSIN II RECEPTOR ANTAGONISTS ON HEART FAILURE}

Angiotensin II receptor antagonist role in heart failure treatment for heart failure makes a lot of progress, but the 5year mortality rate is still around $50 \%$, indicating that prevention is essential to its occurrence. Because RAS activation plays a key role in cardiovascular disease, so using RAS to reduce the risk of cardiovascular disease is reasonable for the idea of drug targets. The guidelines of the US and European reported that ACEI and $\beta$-blocker therapy is the cornerstone of heart failure treatment. RAS inhibitors have been shown to reduce the load on the heart in rats with experimental heart failure, enhance heart function and improve survival and block left ventricular remodeling after myocardial infarction in animal models of myocardial infarction, myocardial structural changes. CHARM-Added trial results showed that on the basis of taking blockers, additional services AT1 receptor antagonist in patients with chronic heart failure can increase cardiac output, further reducing the incidence of myocardial infarction or heart failure.

In the past 10 years, plenty of evidences indicate that angiotensin II receptor antagonist (ARB) can lower blood pressure, block or reverse left ventricular hypertrophy, reduce proteinuria, reduce chronic heart failure, and left ventricular systolic dysfunction after myocardial infarction in patients with then the incidence of cardiovascular disease mortality rates. The role of Ang II is mediated through its receptor, mainly AT1 and AT2. Ang II binding with AT1 can adversely affect vasoconstriction, sodium retention, etc. And the combination of Ang II and AT2 can produce vasodilation, natriuretic and diuresis beneficial effects. ARB because of its high affinity (affinity with AT1), a highly selective (only blocking AT1), high specificity (only affects Ang II receptor), blocks the adverse effects, but does not block the AT2, thus promoting a beneficial effect. Therefore, these drugs have a "double benefit effect".

AT1 receptor antagonist is for the treatment of hypertension ARB initially, and is for the treatment of heart failure later. Currently in the cardiovascular field ARB application refers: orally active, metabolically stable, loyal non-peptide selective AT1 receptor antagonist. After ARB losartan listed first in 1994, in 10 years' time, there are six kinds of ARB used in clinical at least. They are losartan, valsartan, Yip losartan, irbesartan, candesartan Tanzania and telmisartan. Clinical mainly used for the antihypertensive effect. Reducing the role of left ventricular hypertrophy: among patients with hypertension and heart failure, Ang II synthesis is one of the main factors contributing to cardiac hypertrophy, which increases the peripheral resistance, myocardial contractility, promotes water and sodium retention and increases blood pressure, indirectly causing cardiac and vascular wall thickening; Meanwhile the heart and blood vessel walls through autocrine and paracrine non hemodynamic effects directly stimulate the growth of cardiac and vascular smooth muscle cells, leading to cardiac hypertrophy. ARB may antagonize these effects of the AT1 receptor-mediated; inhibit myocardial cell proliferation, delay or reversal of cardiac hypertrophy. In addition there are renal protective effect, cerebral vascular protective effect and anti-tumor effect.

\section{CONCLUSIONS}

The discovery of ACE2 greatly enriched the past view held by people on the renin-angiotensin system. In recent years, both in the study of live animals and vitro cells, ACE2 and its metabolites Ang (1-7) in the pathophysiology of the development of cardiovascular disease should have played the important role. For the regulation of blood pressure, antiatherosclerosis the formation of atherosclerosis and the protection of cardiac function it has an active effect, which laid the theoretical foundation for future clinical application of ACE2 in the treatment of cardiovascular diseases.

\section{References}

[1] Geng Xinjun, Gao Yaoqing, She Minjuan, Chen Ning, Yan Shilin .different grade disease and hypertension in different age renin - correlation between the activity of angiotensin-aldosterone II - [J] Shaanxi Medical Journal, 2011,08: 957 -959.

[2] Du Peige, An Liping, Wang Ailin, Okubo rock male rats three peptidase I renin - angiotensin I, II, III hydrolysis of [J] Basic \& Clinical Medicine, 2006,04: 396-399.

[3] Huang Zewei plasma renin - angiotensin - aldosterone clinical application detection (with 122 cases detected analysis) [J] Fujian Journal of Medicine, 2004,01: 98-100.

[4] Luosu Fen, Jin Xueqing. renin - angiotensin system and the metabolic syndrome research progress [J] Medical Summary, 2009,03: 464-466.

[5] Li Jinglin, Zhao Wencheng, Kuang Fangmei. Propofol combined with sufentanil for laparoscopic surgery patients' renin - angiotensin aldosterone system [J] FOREIGN MEDICAL, 2014, 33: 151 -152.

[6] Li Zhaoliang. Relations elderly life satisfaction and cardiovascular disease [D]. Jilin University, 2004.

[7] Chen Zhuo. Gestational diabetes insulin resistance and cardiovascular disease risk factors correlation of [D]. Kunming Medical College, 2004.

[8] Zhang Feng,Qian Juying.with cardiovascular disease in elderly patients perioperative $[\mathrm{J}]$ Chinese Journal of Practical Surgery, 2009,02: 112-115. 